\title{
Hall Effects on Hydromagnetic Rotating Couette Flow
}

\author{
S. Das \\ Department of Mathematics \\ University of Gour Banga \\ Malda 732 103, India
}

\author{
B.C. Sarkar \\ Department of \\ Applied Mathematics \\ Vidyasagar University \\ Midnapore 721 102, India
}

\author{
R. N. Jana \\ Department of \\ Applied Mathematics \\ Vidyasagar University \\ Midnapore 721 102, India
}

\begin{abstract}
The hydromagnetic Couette flow of a viscous incompressible electrically conducting fluid bounded by two infinite parallel non-conducting plates in the presence of a uniform transverse magnetic field is studied on taking Hall currents into account. The relevant equations are solved analytically. The solution obtained shows that the inclusion of Hall currents gives some interesting results. It is found that the Hall currents tend to retard the primary velocity in the region near the stationary plate and accelerates the primary velocity in the region near the moving plate. It has reverse effect on the secondary velocity in the presence of Hall currents. It is observed that the induced magnetic field components are radically influenced by the Hall currents. On the other hand, the shear stresses at the moving plate decreases with an increase in the Hall parameter. It is interesting to note that both for large squared-Hartmann number and rotation parameter there exists a single-deck boundary layer in the region near the stationary plate. The boundary layer thickness increases with an increase in Hall parameter whereas it decreases with an increase in either squared-Hartmann number or rotation parameter. The asymptotic behavior of the solutions are discussed for large values of squared-Hartmann number and rotation parameter.
\end{abstract}

Keywords: Hydromagnetic, Couette flow, Hall current, Hartmann number, rotation parameter, steady flow and induced magnetic fields.

\section{INTRODUCTION}

MHD Couette flows find widespread applications in geophysics, planetary sciences and also many areas of industrial engineering. For many decades engineers have studied such flows with or without rotation and also for both the steady and unsteady cases. Such studies have entailed many configurations including the flow between rotating plates, rotating concentric cylinders, etc. Hartmann and Lazarus[1] have studied the influence of a transverse uniform magnetic field on the flow of a viscous incompressible electrically conducting fluid between two infinite parallel stationary and insulating plates. The problem is extended in a numerous ways. Closed form solutions for the velocity fields have been obtained [2-5] under different physical effects. In the above mentioned cases the Hall term has been ignored in applying Ohm's law as it has no marked effect for small and moderate values of the magnetic field. However, the current trend for the application of magnetohydrodynamics is that towards a strong magnetic field so that the influence of electromagnetic force is noticeable. Under these conditions, the Hall current is important and it has a marked effect on the magnitude and direction of the current density and consequently on the magnetic force. Tani [6] studied the Hall effects on the steady motion of a viscous incompressible electrically conducting fluid in channel. Jana and Datta $[7,8]$ examined the Hall effects on Couette flow. Soundalgekar et al. $[9,10]$ studied the effects of Hall currents on the steady MHD Couette flow with heat transfer. The temperatures of the two plates are assumed either to be constant or varying linearly along the plates in the direction of the flow. Abo-El-Dahab[11] has studied the effects of Hall currents on the steady Hartmann flow subject to a uniform suction and injection at the bounding plates. The hydromagnetic Couette flow in a rotating system with Hall currents was investigated by Kumar et al.[12]. Mandal and Mandal [13] discussed the effects of Hall currents on MHD Couette flow between thick arbitrarily conducting plates in a rotating system. The steady hydromagnetic Couette flow in a rotating system with non-conducting walls was presented by Seth and Singh [14]. Seth et al.[15] investigated the Hall effects on oscillatory hydromagnetic Couette flow in a rotating system. Mandal et al.[16] presented an analysis on combined effects of Coriolis force and Hall currents on a steady MHD Couette flow and heat transfer. Combined effects of Hall and ion-slip currents on unsteady MHD Couette flow in a rotating system have been investigated by Jha and Apere[17]. An exact solution of an oscillatory MHD flow through a porous medium bounded by rotating porous channel in the presence of Hall currents have been obtained by Singh and Kumar[18]. Chauhan and Rastogi [20] have examined the heat transfer effects on rotating MHD Couette flow in a channel partially filled by a porous medium with Hall current. Ghosh and Bhattacharjee [21] have examined the Hall effects on a steady hydromagnetic flow in a rotating channel in the presence of an inclined magnetic field. Ghosh [22] has discussed the effects of Hall currents on MHD Couette flow in a rotating system with an arbitrary magnetic field. Beg et al.[23] have described the unsteady magnetohydrodynamic Hartmann-Couette flow and heat transfer in a Darcian fluid with Hall currents, ion-slip, viscous and Joule heating effects. Recently, Das et al. [24] and Maji et al. [25] have studied the Hall effects on MHD Couette flow in a rotating system. The combined effects of Hall currents and rotation on steady hydromagnetic Couette flow have been examined by Sarkar et al. [26].

The objective of the present paper is to investigate the influences of Hall currents on the steady hydromagnetic Couette flow of a viscous incompressible electrically conducting fluid in a rotating system. The fluid is flowing between two electrically insulating plates. The upper plate is moving with a constant velocity $U_{0}$ while the lower plate is kept stationary. An external uniform magnetic field is applied perpendicular to the plates and the Hall effects are taken into consideration. Effects of governing parameters on the fluid velocity components, the induced magnetic field components and the shear stresses at the plates are presented graphically.

\section{MATHEMATICAL FORMULATION AND ITS SOLUTION}

The basic equations of magnetohydrodynamics for steady flow are

$$
(\vec{q} \cdot \nabla) \vec{q}+2 \Omega \hat{j} \times \vec{q}=-\frac{1}{\rho} \nabla p+\nu \nabla^{2} \vec{q}+\frac{1}{\rho} \vec{j} \times \vec{B},
$$




$$
\begin{aligned}
& \nabla \cdot \vec{q}=0, \\
& \nabla \times \vec{B}=\mu_{e} \vec{j}, \\
& \nabla \times \vec{E}=0 \quad \text { (for steady flow), } \\
& \nabla \cdot \vec{B}=0,
\end{aligned}
$$

together with generalized Ohm's law taking Hall current into account is

$$
\vec{j}+\frac{\omega_{e} \tau_{e}}{B_{0}}(\vec{j} \times \vec{B})=\sigma(\vec{E}+\vec{q} \times \vec{B}),
$$

where $\vec{q}, \vec{B}, \vec{E}, \vec{j}$ are respectively the velocity vector, the magnetic field vector, the electric field vector and the current density vector. Also $\sigma, v, \mu_{e}, \rho, B_{0}, \omega_{e}$ and $\tau_{e}$ are respectively the electrically conductivity, kinematic viscosity, magnetic permeability, fluid density, applied magnetic field, cyclotron frequency and electron collision time. In writing the equation (6) the ion slip and thermoelectric effects as well as the electron pressure gradient are neglected.

Consider the viscous incompressible electrically conducting fluid bounded by two infinitely long horizontal parallel plates separated by a distance $d$. Choose a Cartesian co-ordinate system with $x$-axis along the lower stationary plate in the direction of the flow, the $y$-axis is normal to the plates and the $z$-axis is perpendicular to $x y$-plane(see Fig.1). The plates are assumed to be electrically non-conducting. The upper plate is moving with a constant velocity $U_{0}$ while the lower plate is held at rest. The plates and the fluid rotate in unison with uniform angular velocity $\Omega$ about an axis perpendicular to the plates. A uniform magnetic field $B_{0}$ is applied in the positive $y$-direction. The Hall effects are taken into consideration and consequently a $z$-component for the velocity and magnetic fields are expected to arise. The velocity components are $(u, v, w)$ relative to a frame of reference. Since the plates are infinitely long, all physical variables, except pressure, depend on $y$ only.

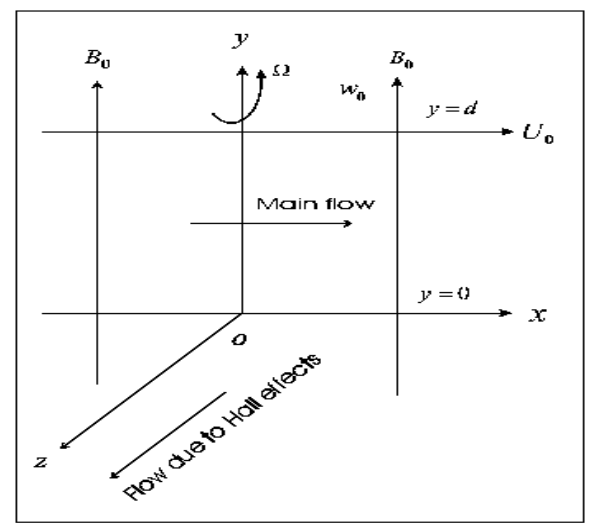

\section{Fig.1: Geometry of the Problem}

The equation of continuity $\nabla \cdot \vec{q}=0$ with no-slip condition at the plates gives $v=0$ everywhere in the flow where $\vec{q} \equiv(u, v, w)$. The solenoidal equation $\nabla \cdot \vec{B}=0$ gives $B_{y}=$ constant $=B_{0} \quad$ everywhere in the flow, where $\vec{B} \equiv\left(B_{x}, B_{y}, B_{z}\right)$.

The momentum equations for the fully developed steady flow are

$$
\begin{aligned}
& 2 \Omega w=-\frac{1}{\rho} \frac{\partial p}{\partial x}+v \frac{d^{2} u}{d y^{2}}+\frac{B_{0}}{\rho \mu_{e}} \frac{d B_{x}}{d y}, \\
& 0=\frac{1}{\rho} \frac{\partial p}{\partial y}+\frac{1}{2 \rho \mu_{e}}\left(\frac{d B_{x}^{2}}{d y}+\frac{d B_{z}^{2}}{d y}\right), \\
& -2 \Omega u=-\frac{1}{\rho} \frac{\partial p}{\partial z}+v \frac{d^{2} w}{d y^{2}}+\frac{B_{0}}{\rho \mu_{e}} \frac{d B_{z}}{d y},
\end{aligned}
$$

where $p$ is the fluid pressure.

Eliminating $\vec{E}$ from equations (4) and (6), we have the $x$ - and $z$-components of the magnetic induction equations as

$$
\begin{aligned}
& -\frac{d^{2} B_{x}}{d y^{2}}+m \frac{d^{2} B_{z}}{d y^{2}}=\sigma \mu_{e} B_{0} \frac{d u}{d y}, \\
& -\frac{d^{2} B_{z}}{d y^{2}}-m \frac{d^{2} B_{x}}{d y^{2}}=\sigma \mu_{e} B_{0} \frac{d w}{d y},
\end{aligned}
$$

where $m=\omega_{e} \tau_{e}$ is the Hall parameter.

The boundary conditions for the velocities and the magnetic fields are

$$
\begin{aligned}
& u=0, w=0, B_{x}=0, B_{z}=0 \text { at } y=0, \\
& u=U_{0}, w=0, B_{x}=0, B_{z}=0 \text { at } y=d .
\end{aligned}
$$

On the use of the boundary condition at $y=d$, we have from equations (7) and (9)

$$
-\frac{1}{\rho} \frac{\partial p}{\partial x}=0 \text { and }-\frac{1}{\rho} \frac{\partial p}{\partial z}=-2 \Omega U_{0} .
$$

On the use of (13), equations (7) and (9) become

$$
\begin{aligned}
& 2 \Omega w=v \frac{d^{2} u}{d y^{2}}+\frac{B_{0}}{\rho \mu_{e}} \frac{d B_{x}}{d y}, \\
& -2 \Omega\left(u-U_{0}\right)=v \frac{d^{2} w}{d y^{2}}+\frac{B_{0}}{\rho \mu_{e}} \frac{d B_{z}}{d y} .
\end{aligned}
$$

Introducing the non-dimensional variables

$$
\eta=\frac{y}{d},\left(u_{1}, w_{1}\right)=\frac{(u, w)}{U_{0}},\left(b_{x}, b_{z}\right)=\frac{\left(B_{x}, B_{z}\right)}{\sigma \mu_{e} B_{0} U_{0} d},
$$

equations (14), (15), (10) and (11) become

$$
\begin{aligned}
& 2 K^{2} w_{1}=\frac{d^{2} u_{1}}{d \eta^{2}}+M^{2} \frac{d b_{x}}{d \eta}, \\
& -2 K^{2}\left(u_{1}-1\right)=\frac{d^{2} w_{1}}{d \eta^{2}}+M^{2} \frac{d b_{z}}{d \eta}, \\
& -\frac{d^{2} b_{x}}{d \eta^{2}}+m \frac{d^{2} b_{z}}{d \eta^{2}}=\frac{d u_{1}}{d \eta}, \\
& -\frac{d^{2} b_{z}}{d \eta^{2}}-m \frac{d^{2} b_{x}}{d \eta^{2}}=\frac{d w_{1}}{d \eta},
\end{aligned}
$$

where $\quad M=B_{0} d\left(\frac{\sigma}{\rho v}\right)^{1 / 2}$ is the Hartmann number and $K^{2}=\frac{\Omega d^{2}}{v}$ the rotation parameter.

Equations (17) and (18) and equations (19) and (20) can be combined into the following equations

$$
\begin{aligned}
& \frac{d^{2} F}{d \eta^{2}}+M^{2} \frac{d b}{d \eta}+2 i K^{2} F=2 i K^{2}, \\
& (1+i m) \frac{d^{2} b}{d \eta^{2}}+\frac{d F}{d \eta}=0,
\end{aligned}
$$

where

$$
F=u_{1}+i w_{1}, b=b_{x}+i b_{z} \text { and } i=\sqrt{-1} .
$$

The corresponding boundary conditions for $F(\eta)$ and $b(\eta)$ are $F=0$ at $\eta=0$ and $F=1$ at $\eta=1$, 


$$
b=0 \text { at } \eta=0 \text { and } b=0 \text { at } \eta=1 .
$$

The solutions of the equations (21) and (22) subject to the boundary conditions (24) are

$$
\begin{aligned}
F(\eta) & =\frac{1}{\lambda}[A \sinh \lambda \eta-B(1-\cosh \lambda \eta)], \\
(1+i m) b(\eta) & =\frac{1}{\lambda^{2}}[A(1-\cosh \lambda \eta)-B \sinh \lambda \eta \\
& \left.+\lambda(B+\lambda) \frac{2 i K^{2}(1+i m) \eta}{M^{2}}\right],
\end{aligned}
$$

where

$$
\begin{gathered}
\lambda=\alpha-i \beta \\
\alpha, \beta=\frac{1}{\sqrt{2}}\left[\left\{\left(\frac{M^{2}}{1+m^{2}}\right)^{2}+\left(2 K^{2}+\frac{m M^{2}}{1+m^{2}}\right)^{2}\right\}^{\frac{1}{2}} \pm\left(\frac{M^{2}}{1+m^{2}}\right)\right]^{\frac{1}{2}}, \\
A=\frac{\lambda}{2}\left[\frac{2 i \lambda K^{2} \cosh \lambda-\frac{M^{2}}{1+i m} \sinh \lambda}{\left.i \lambda K^{2} \sinh \lambda+\frac{M^{2}}{1+i m}(1-\cosh \lambda)\right]}\right. \\
B=-\frac{\lambda}{2}\left[\frac{2 i \lambda K^{2} \sinh \lambda+\frac{M^{2}}{1+i m}(1-\cosh \lambda)}{i \lambda K^{2} \sinh \lambda+\frac{M^{2}}{1+i m}(1-\cosh \lambda)}\right]
\end{gathered}
$$

On separating into a real and imaginary parts one can easily obtain the velocity components $u_{1}$ and $w_{1}$ from equation (25) and the induced magnetic field components $b_{x}$ and $b_{z}$ from the equation (26). If $m=0$, then above equations (25) and (26) are identical with the equations (23) and (24) of Seth and Singh[14]

\section{RESULTS AND DISCUSSION}

We have presented the non-dimensional velocity components and induced magnetic field components for several values of squared-Hartmann number $M^{2}$, rotation parameter $K^{2}$ and Hall parameter $m$ in Figs.2-7. Fig.2 shows that the primary velocity $u_{1}$ increases near the stationary plate and then it decreases away from the stationary plate with an increase in squared-Hartmann number $M^{2}$ whereas the magnitude of secondary velocity $w_{1}$ decreases with an increase in $M^{2}$. It reveals that magnetic field tends to accelerate the primary velocity in the region near the stationary plate while it has the reverse effect on the primary velocity in the region near the moving plate and it tends to decelerate the secondary velocity. Fig.3 displays that the primary velocity $u_{1}$ increases with an increase in rotation parameter $K^{2}$ while the magnitude of secondary velocity $w_{1}$ increases in the region near the stationary plate and decreases in the region near the moving plate with an increase in $K^{2}$. The rotation parameter $K^{2}$ defines the relative magnitude of the Coriolis force and the viscous force in the regime, therefore it is clear that high magnitude Coriolis forces are counter-productive for the primary flow. It is seen from Fig.4 that the primary velocity $u_{1}$ decreases near the stationary plate and then it increases away from the stationary plate with an increase in Hall parameter $m$ whereas the magnitude of secondary velocity $w_{1}$ increases in the region near the stationary plate and decreases in the region near the moving plate with an increase in $m$. This implies that Hall parameter tends to decelerate the primary velocity in the region near the stationary plate while it has the reverse effect on the primary velocity in the region near the moving plate. On the other hand, Hall currents tend to accelerate the secondary velocity in the region near the stationary plate while it has the reverse effect on the secondary velocity in the region near the moving plate.

When flow of a conducting fluid is under the influence of a magnetic field, we know that there is a coupling between the flow field and the magnetic field. From physical considerations it is known that the lines of force representing an applied magnetic field influence the fluid flow, which in turn influences these magnetic lines as well. Thus the parameters which influence the flow field in turn influence similarly the induced magnetic fields in the primary and secondary flow directions. Profiles for induced magnetic field $b_{x}$ in the primary flow direction and $b_{z}$ in the secondary flow direction are presented in Figs. 5-7 respectively for various values of the pertinent parameters. It is observed from Fig.5 that both the primary induced magnetic field $b_{x}$ and the secondary induced magnetic field $b_{z}$ decrease with an increase in squared-Hartmann number $M^{2}$. Thus the magnetic field has tendency to reduce both the primary and secondary induced magnetic fields. Fig.6 reveals that the primary induced magnetic field $b_{x}$ increases in the region near the stationary plate and decreases in the region near the moving plate with an increase in rotation parameter $K^{2}$ whereas the magnitude of the secondary induced magnetic field $b_{z}$ decreases with an increase in $K^{2}$. This implies that rotation tends to enhance primary induced magnetic field in the region near the stationary plate and it has reverse effect on the primary induced magnetic field in the region near the moving plate. Rotation tends to reduce the magnitude of the secondary induced magnetic field. It is observed from Fig.7 that with an increase in Hall parameter $m$ the primary induced magnetic field $b_{x}$ decreases whereas the magnitude of secondary induced magnetic field $b_{z}$ increases. When $m=0$, it is negative only in the near region of the upper moving plate and positive in the remaining channel. As $m$ increases it is seen that the magnitude of $b_{z}$ increases significantly in the upper part becomes zero at certain $\eta$ and becomes positive in the lower remaining part of the channel. Thus the Hall parameter has tendency to reduce the primary induced magnetic field and it has reverse effect on the secondary induced magnetic field.

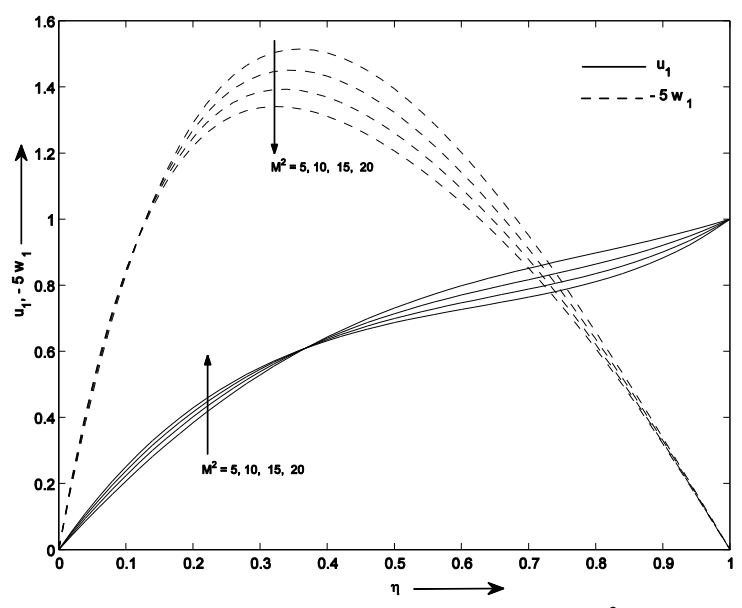

Fig.2: Primary and secondary velocities for $M^{2}$ when $m=0.5$ and $K^{2}=4$ 


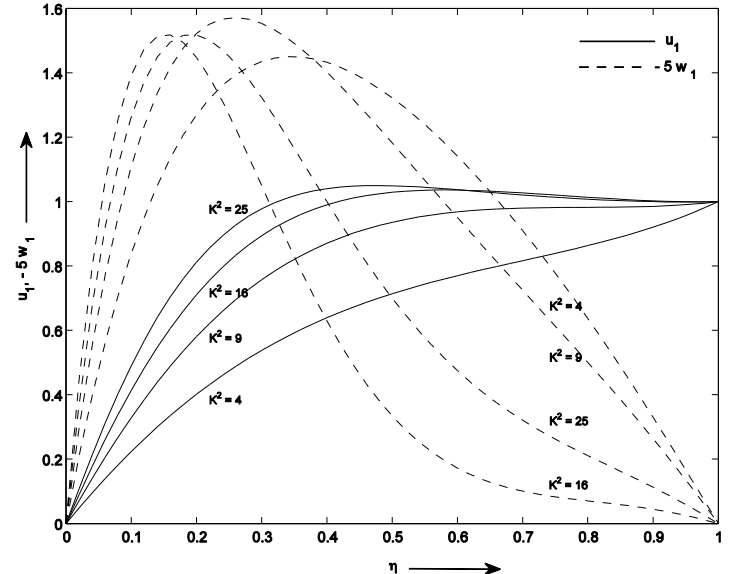

Fig.3: Primary and secondary velocities for $K^{2}$ when $M^{2}=10$ and $m=0.5$

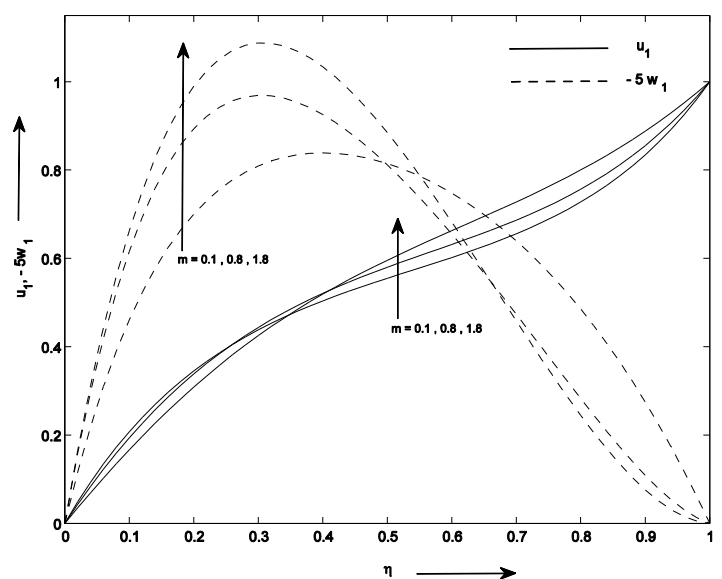

Fig.4: Primary and secondary velocities for $m$ when $M^{2}=20$ and $K^{2}=2$

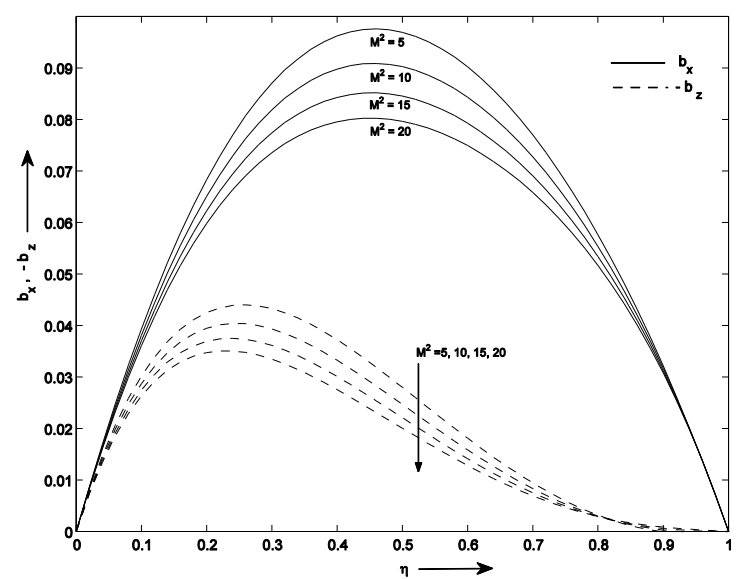

Fig.5: Primary and secondary magnetic fields for $M^{2}$ when $m=0.5$ and $K^{2}=4$

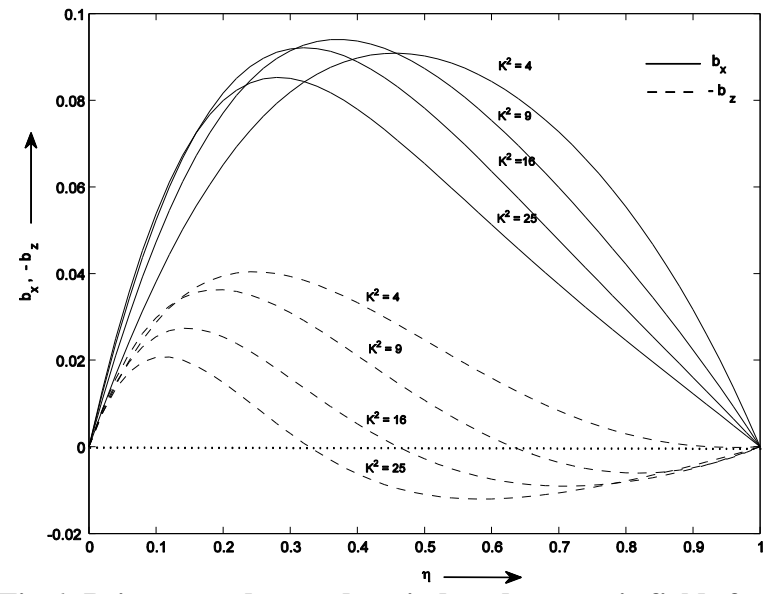

Fig.6: Primary and secondary induced magnetic fields for $K^{2}$ when $M^{2}=10$ and $K^{2}=4$

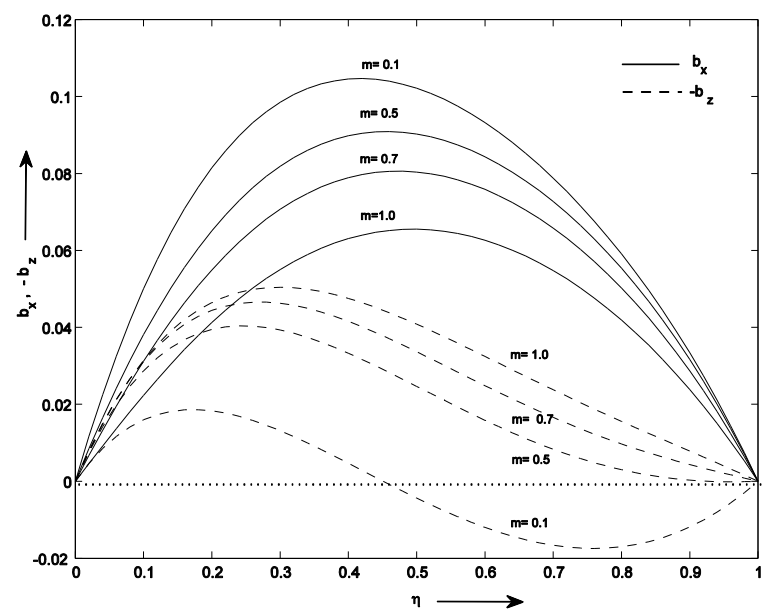

Fig.7: Primary and secondary induced magnetic fields for $\boldsymbol{m}$ when $M^{2}=10$ and $K^{2}=4$

The non-dimensional shear stresses due to the primary and secondary flows at the stationary plate $(\eta=0)$ and the moving plate $(\eta=1)$ are respectively

$$
\begin{aligned}
& \tau_{x_{0}}+i \tau_{z_{0}}=\left(\frac{d F}{d \eta}\right)_{\eta=0} \\
& =\frac{\lambda}{2}\left[\frac{2 i \lambda K^{2} \cosh \lambda-\frac{M^{2}}{1+i m} \sinh \lambda}{i \lambda K^{2} \sinh \lambda+\frac{M^{2}}{1+i m}(1-\cosh \lambda)}\right], \\
& \tau_{x_{1}}+i \tau_{z_{1}}=\left(\frac{d F}{d \eta}\right)_{\eta=1} \\
& =\frac{\lambda}{2}\left[\frac{2 i \lambda K^{2}-\frac{M^{2}}{1+i m} \sinh \lambda}{i \lambda K^{2} \sinh \lambda+\frac{M^{2}}{1+i m}(1-\cosh \lambda)}\right],
\end{aligned}
$$

where $\lambda$ is given by (27)

Numerical results of the shear stresses at the stationary plate $(\eta=0)$ and moving $(\eta=1)$ are depicted in Figs.8-11 against $m$ for various values of $M^{2}$ and $K^{2}$. Fig. 8 shows that both the primary shear stress at the stationary plate $\tau_{x_{0}}$ and the magnitude of secondary shear stress at the stationary plate $\tau_{z_{0}}$ increase with an increase in squared-Hartmann number $M^{2}$. 
This implies that the magnetic field has tendency to increase the primary shear stress as well as the secondary shear stress at the stationary plate. An increase in Hall parameter $m$ leads to decrease in the shear stress $\tau_{x_{0}}$ and increase in the magnitude of $\tau_{z_{0}}$ for fixed value of $M^{2}$. Thus the Hall parameter has tendency to reduce the primary shear stress and it has reverse effect on the secondary shear stress at the stationary plate. It is seen from Fig. 9 that both the primary shear stress $\tau_{x_{0}}$ and the magnitude of the secondary shear stress $\tau_{z_{0}}$ increase with an increase in rotation parameter $K^{2}$. This implies that rotation has a tendency to enhance primary as well as the secondary shear stresses at the stationary plate. Fig.10 reveals that the primary shear stress at the moving plate $\tau_{x_{1}}$ increases with an increase in squared-Hartmann number $M^{2}$ whereas the secondary shear stress at the moving plate $\tau_{z_{1}}$ first increases, reaches a maximum and then decreases with an increase in $M^{2}$ . It is seen from Fig.11 that the primary shear stress $\tau_{x_{1}}$ decreases with an increase in either rotation parameter $K^{2}$ or Hall parameter $m$ whereas the secondary shear stress $\tau_{z_{1}}$ increases with an increase in rotation parameter $K^{2}$ and it decreases with an increase in Hall parameter $m$. Thus the Hall parameter has tendency to reduce the primary shear stress as well as the secondary shear stress at the moving plate.

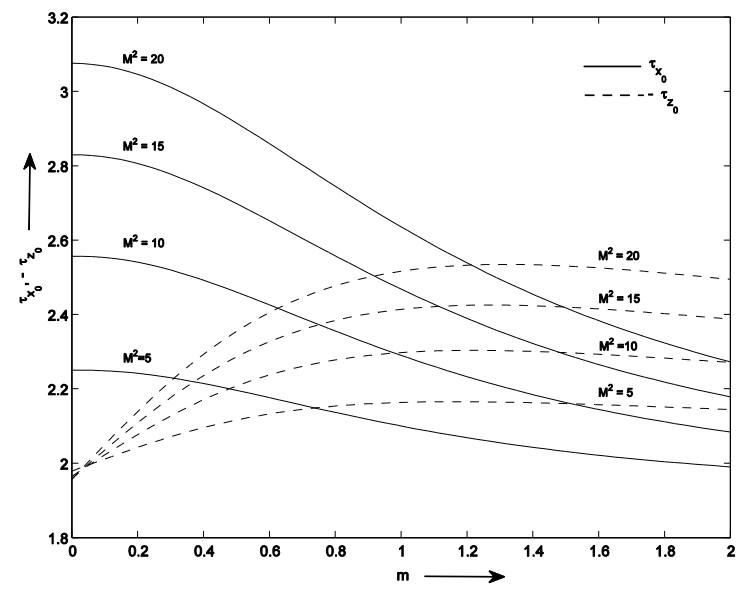

Fig.8: Primary and secondary shear stresses at stationary plate for $M^{2}$ when $K^{2}=4$

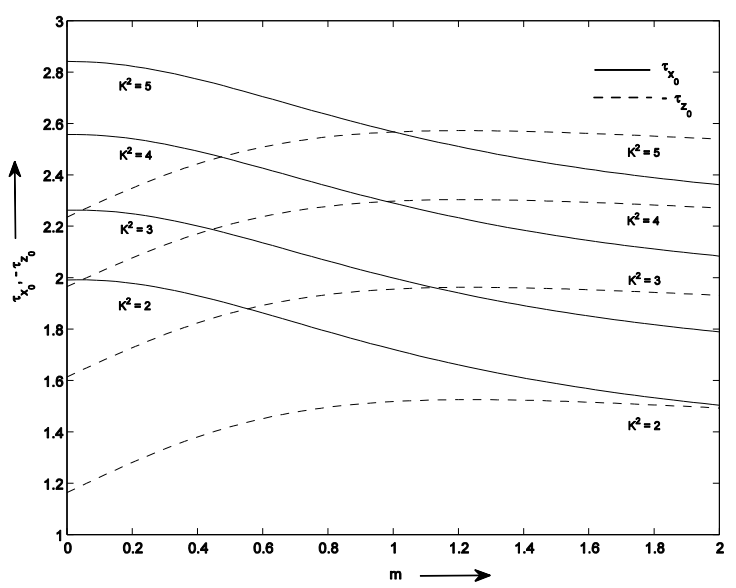

Fig.9: Primary and secondary shear stresses at stationary plate for $K^{2}$ when $M^{2}=10$

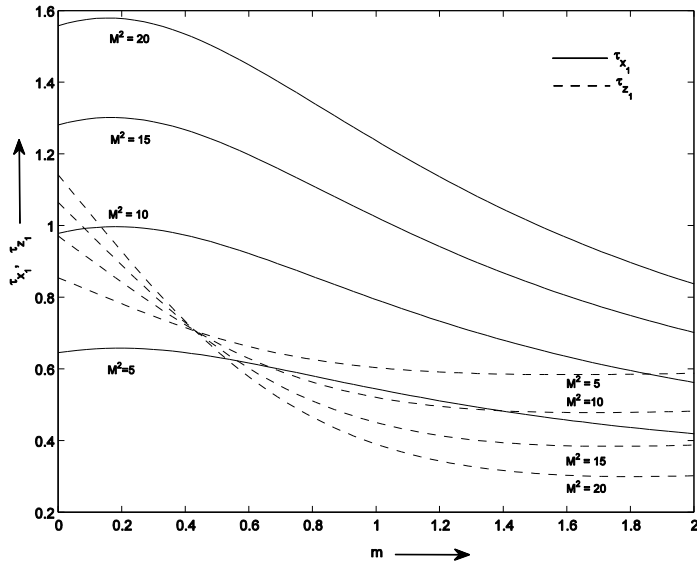

Fig.10: Primary and secondary shear stresses at moving plate for $M^{2}$ when $K^{2}=4$

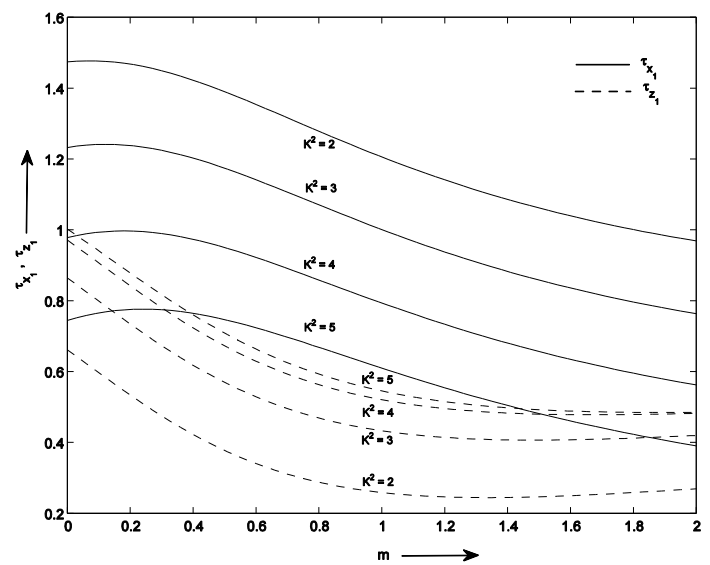

Fig.11: Primary and secondary shear stresses at moving plate for $K^{2}$ when $M^{2}=10$

We shall now discuss the asymptotic behavior of the solutions (25) and (26) for large values of $M^{2}$ and $K^{2}$ :

Case I: When $K^{2} \square 1$ and $M^{2} \square O(1)$, then the equations (21) and (22) give

$$
\begin{aligned}
& u_{1}=1-e^{-\alpha_{1} \eta} \cos \beta_{1} \eta, \\
& w_{1}=-e^{-\alpha_{1} \eta} \sin \beta_{1} \eta, \\
& b_{x}=\frac{1}{2 K\left(1+m^{2}\right)}[(1+m) \\
& \left.\quad-e^{-\alpha_{1} \eta}\left\{(1+m) \cos \beta_{1} \eta-(1-m) \sin \beta_{1} \eta\right\}\right], \\
& b_{z}=\frac{1}{2 K\left(1+m^{2}\right)}[(1-m) \\
& \left.-e^{-\alpha_{1} \eta}\left\{(1-m) \cos \beta_{1} \eta+(1+m) \sin \beta_{1} \eta\right\}\right],
\end{aligned}
$$

where

$$
\alpha_{1}, \beta_{1}=K\left[1 \pm \frac{(1 \pm m) M^{2}}{4 K^{2}\left(1+m^{2}\right)}\right] .
$$

It is seen from equations (33) and (34) that there exists a singledeck boundary layer of thickness of the order $\mathrm{O}\left(1 / \alpha_{1}\right)$ where $\alpha_{1}$ is given by (37). It is seen that the thickness of this boundary layer increases with an increase in Hall parameter $m$ and decreases with an increase in either rotation parameter $K^{2}$ or squared-Hartmann $M^{2}$.

Case II: When $M^{2} \square 1$ and $K^{2} \square O(1)$, then equations (21) 


$$
\begin{aligned}
& \text { and (22) give } \\
& u_{1}=\frac{1}{2}\left[1-e^{-\alpha_{2} \eta}\left(\cos \beta_{2} \eta+\frac{K^{2} \sqrt{1+m^{2}}}{M} \sin \beta_{2} \eta\right)\right. \text {, } \\
& w_{1}=-\frac{1}{2}\left[\frac{K^{2} \sqrt{1+m^{2}}}{M}+e^{-\alpha_{2} \eta}\left(\sin \beta_{2} \eta-\frac{K^{2} \sqrt{1+m^{2}}}{M} \cos \beta_{2} \eta\right)\right. \text {, } \\
& b_{x}=\frac{1}{2}\left[\frac { 1 } { M \sqrt { 1 + m ^ { 2 } } } \left\{\left(1+\frac{1}{2} m^{2}\right)-e^{-\alpha_{2} \eta}\left(\left(1+\frac{1}{2} m^{2}\right) \cos \beta_{2} \eta\right.\right.\right. \\
& \left.\left.\left.+\left(\frac{K^{2}\left(1+m^{2}\right)}{M}+\frac{1}{2} m\right) \sin \beta_{2} \eta\right)\right\}\right] \text {, } \\
& b_{z}=\frac{1}{2}\left[\frac{2 K^{2} \eta}{M^{2}}-\frac{1}{M \sqrt{1+m^{2}}}\left\{\frac{1}{2} m+\frac{K^{2}\left(1+m^{2}\right)}{M}\right.\right. \\
& +e^{-\alpha_{2} \eta}\left(\left(1+\frac{1}{2} m^{2}\right) \sin \beta_{2} \eta\right. \\
& \left.\left.\left.-\left(\frac{K^{2}\left(1+m^{2}\right)}{M}+\frac{1}{2} m\right) \cos \beta_{2} \eta\right)\right\}\right] \text {, }
\end{aligned}
$$

where

$$
\alpha_{2}=\frac{M}{\sqrt{1+m^{2}}}, \beta_{2}=\left(\frac{K^{2}}{M} \sqrt{1+m^{2}}+\frac{m M}{2 \sqrt{1+m^{2}}}\right) .
$$

It is seen from equations (38) and (39) that there exists a singledeck boundary layer of thickness of the order $\mathrm{O}\left(1 / \alpha_{2}\right)$ where $\alpha_{2}$ is given by (42). It is seen that the thickness of this boundary layer increases with increase in Hall parameter $m$ while it decreases with an increase in Hartmann number $M$. It is interesting to note that for large Hartmann number, that is, for strong magnetic field the boundary layer thickness is independent of rotation parameter $K^{2}$.

\section{CONCLUSION}

An investigation of the effects of Hall currents and rotation on the hydromagnetic Couette flow of a viscous incompressible electrically conducting fluid in the presence of a uniform transverse magnetic field has been carried out. Hall currents stifle the primary flow in the channel while the secondary flow responds more positively with it. The secondary flow is due to Hall effects and rotation, hence both effects mark significantly on the secondary flow throughout the channel. Rotation has significant influences on the velocity components. Hall currents have marked effects on the induced magnetic field. The magnetic field and rotation have tendency to enhance the primary and secondary shear stresses at the stationary plate. Hall currents tends to reduce the primary shear stress and it has reverse effect on the secondary shear stress at the stationary plate. There exists a single-deck boundary layer in the region near the stationary plate either for large squared-Hartmann number or rotation parameter. The boundary layer thickness increases with an increase in Hall parameter whereas it decreases with an increase in either squared-Hartmann number or rotation parameter. The present study concerning magnetohydrodynamics of rotating fluids in the presence of Hall currents has immediate relevance to many aspects of fluid engineering, astrophysics and geophysics and therefore has wide applications in these areas.

\section{REFERENCES}

[1] Hartmann, J. and Lazarus, F.(1937). Kgl. Danske Videnskab. Selskab. Mat.-Fys. Medd., 15(6- 7) .
[2] Tao, L.N.(1960). Magnetohydrodynamic effects on the formation of Couette flow, J. Aerospace Sci., $27: 334$.

[3] Alpher, R.A.(1961). Heat transfer in magnetohydrodynamic flow between parallel plates, Int. J. Heat and Mass Transfer, $3: 108$.

[4] Sutton, G.W. and Sherman, A.(1965). Engineering Magnetohydrodynamics. McGraw-Hill .

[5] Cramer, K. and Pai, S.(1973). Magnetofluid dynamics for engineers and applied physicists. McGraw-Hill .

[6] Tani, I.(1962). Steady motion of conducting fluids in channels under transverse magnetic fields with consideration of Hall effect, J. Aerospace Sci., 29 :287.

[7] Jana, R.N. and Datta, N. (1977). Hall effects on unsteady Couette flow, Int. J. Engg. Sci., 15:35-43.

[8] Jana, R.N. and Datta, N. (1980). Hall effects on MHD Couette flow in a rotating of system, Czech. J. Physics, 30: 659-667.

[9] Soundalgekar, V. M., Vighnesam, N.V. and Takhar, H.S.(1979), Hall and ion-slip effects in MHD Couette flow with heat transfer, IEEE Transactions on Plasma Sciences, PS-7(3) .

[10] Soundalgekar, V.M. and Uplekar, A.G.(1986), Hall effects in MHD Couette flow with heat transfer. IEEE Transactions on Plasma Science, PS-14(5).

[11] Abo-El-Dahab, E.M.H.(1993). Effect of Hall currents on some magnetohydrodynamic flow problems, Master Thesis, Dept.Math., Fac. of Science, Helwan Univ., Egypt.

[12] Kumar, A., Seth, G. S. and Talib, A.(2007) Hydromagnetic Couette flow in a rotating system with Hall effects, Acta Ciencia Indica, 33M: 937.

[13] Mandal, G. and Mandal, K. K.(1983), Effects of Hall current on MHD Couette flow between thick arbitrarily conducting plates in a rotating system, J. Phys. Soc. Jpn, $52: 470$.

[14] Seth, G. S. and Singh, J.K. (2011), Steady hydromagnetic Couette flow in a rotating system with non-conducting walls, Int. J. Eng. Sci. Tech., 3(2) : 146-156.

[15] Seth, G. S., Nandkeolyar, R. and Ansari, Md. S.(2009), Hall effects on oscillatory hydromagnetic Couette flow in a rotating system, Int. J. Acad. Res., 1:6-17.

[16] Mandal, G., Mandal, K. K., Choudhury, G.(1982). On combined effects of Coriolis force and Hall current on steady MHD Couette flow and heat transfer, Phys. Soc.Jpn, J., 51: 2010-2015.

[17] Jha, B.K. and Apere, C. A.(2010). Combined Effect of Hall and Ion-Slip Currents on unsteady MHD Couette flows in a rotating system, J. Phys. Soc. Jpn., 79:104401.

[18] Singh, K.D. and Kumar, R. (2010). An exact solution of an oscillatory MHD flow through a porous medium bounded by rotating porous channel in the presence of Hall current, Int. J. Applied Math. Mech., 6(13):28-40.

[19] Attia, H.A.(2011), Steady MHD Couette flow with temperature-dependent physical properties, Archive Of Applied Mechanics, 75(4-5):268-274.

[20] Chauhan, D.S. and Rastogi, P. (2012). Heat transfer effects on rotating MHD Couette flow in a channel partially filled 
by a porous medium with Hall current, J. Applied Science and Engineering, 15(3): 281-290.

[21] Ghosh, S. K. and Bhattacharjee, P. K. (2000). Hall effects on steady hydromagnetic flow in a rotating channel in the presence of an inclined magnetic field, Chech. J. Phys., 50: 759-767.

[22] Ghosh, S. K.(2002). Effects of Hall current on MHD Couette flow in a rotating system with arbitrary magnetic field, Czech. J. Phys., 52: 51-63.

[23] Beg, O. A. Zueco, J. and Takhar, H. S.(2009). Unsteady magnetohydrodynamic Hartmann-Couette flow and heat transfer in a Darcian channel with Hall current, ionslip, viscous and Joule heating effects: network numerical solutions, Communications in Non-linear Science and
Numerical Simulation, 14(4): 1082-1097.

[24] Das, S., Sarkar, B. C. and Jana, R. N. (2011). Hall effects on MHD Couette flow in a rotating system, Int. J. Computer Applications, 35(13):22-30.

[25] Maji, S.L., Das, S. and Jana, R. N. (2013). Hall effects on unsteady Couette flow in a rotating system, Journal of Mechanics, 29(03): 443-452.

[26] Sarkar, B. C., Das, S. and Jana, R. N. (2013). Combined effects of Hall currents and rotation on steady hydromagnetic Couette flow, Research J. Applied Sciences, Engineering and Technology, 5(6): 1864-1875. 